\title{
Simultaneous measurement of time-domain fNIRS and physiological signals during a cognitive task
}

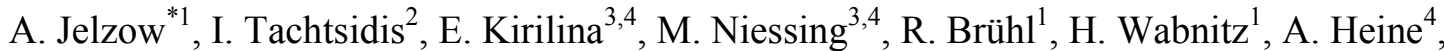 \\ B. Ittermann ${ }^{1}$, R. Macdonald ${ }^{1}$ \\ ${ }^{1}$ Physikalisch-Technische Bundesanstalt (PTB), Abbestr. 2-12, 10587 Berlin, Germany \\ ${ }^{2}$ Dept. Medical Physics and Bioengineering, Biomedical Optics Laboratory, University College \\ London, Gower Street, London, UK \\ ${ }^{3}$ Dahlem Institute of Neuroimaging of Emotion, Berlin, Germany \\ ${ }^{4}$ Dept. of Education and Psychology, Free University of Berlin, Berlin, Germany \\ *alexander.jelzow@ptb.de, phone+49 3034817954
}

\begin{abstract}
Functional near-infrared spectroscopy (fNIRS) is a commonly used technique to measure the cerebral vascular response related to brain activation. It is known that systemic physiological processes, either independent or correlated with the stimulation task, can influence the optical signal making its interpretation challenging. The aim of the present work is to investigate the impact of task-evoked changes in the systemic physiology on fNIRS measurements for a cognitive paradigm. For this purpose we carried out simultaneous measurements of time-domain fNIRS on the forehead and systemic physiological signals, i.e. mean blood pressure, heart rate, respiration, galvanic skin response, scalp blood flow (flux) and red blood cell (RBC) concentration changes. We performed measurements on 15 healthy volunteers during a semantic continuous performance task (CPT). The optical data was analyzed in terms of depth-selective moments of distributions of times of flight of photons through the tissue. In addition, cerebral activation was localized by a subsequent fMRI experiment on the same subject population using the same task. We observed strong non-cerebral taskevoked changes in concentration changes of oxygenated hemoglobin in the forehead. We investigated the temporal behavior and mutual correlations between hemoglobin changes and the systemic processes. Mean blood pressure (BP), galvanic skin response (GSR) and heart rate exhibited significant changes during the activation period, whereby BP and GSR showed the highest correlation with optical measurements.
\end{abstract}

Keywords: time-domain NIRS, functional brain activation, BA10, physiological signals, systemic effects

\section{INTRODUCTION}

Functional near-infrared spectroscopy (fNIRS) is often used to measure task-evoked changes in cerebral hemodynamics in the human brain. However, almost any cognitive process is accompanied by changes in systemic physiological signals. It was found that fluctuations in some of the systemic physiological parameters such as mean blood pressure (BP) and heart rate (HR) interfere with the cerebral hemodynamic response as measured by fNIRS [1]. These systemic changes also contribute to the noise of the fNIRS signals and possibly, in some subjects, mask actual brain activation and lead to false positives in brain activation maps [2]. The aim of this study is to investigate the influence of task-evoked systemic physiological parameters on fNIRS measurements of cerebral activation. To achieve this goal we applied timedomain fNIRS (td-fNIRS) on the forehead in combination with simultaneous recordings of mean blood pressure (BP), electrocardiogram (ECG), respiration, galvanic skin response (GSR), scalp blood flow (SBF), and scalp red blood cells (RBC) concentration changes. In order to separate cortical and extracranial hemodynamics we estimated the task-evoked changes in oxy- and deoxyhemoglobin concentration in the forehead using (i) the light attenuation changes (similar to continuous-wave fNIRS recordings) and (ii) the changes in the variance of the distribution of times of flight photons. In addition, we performed a subsequent fMRI experiment on the same subject population using the same task in order to localize the cerebral activation.

Diffuse Optical Imaging III, edited by Andreas H. Hielscher, Paola Taroni, Proc. of SPIE-OSA Biomedical Optics, SPIE Vol. 8088, 808803 · C 2011 SPIE-OSA · CCC code: 1605-7422/11/\$18 · doi: 10.1117/12.889484 


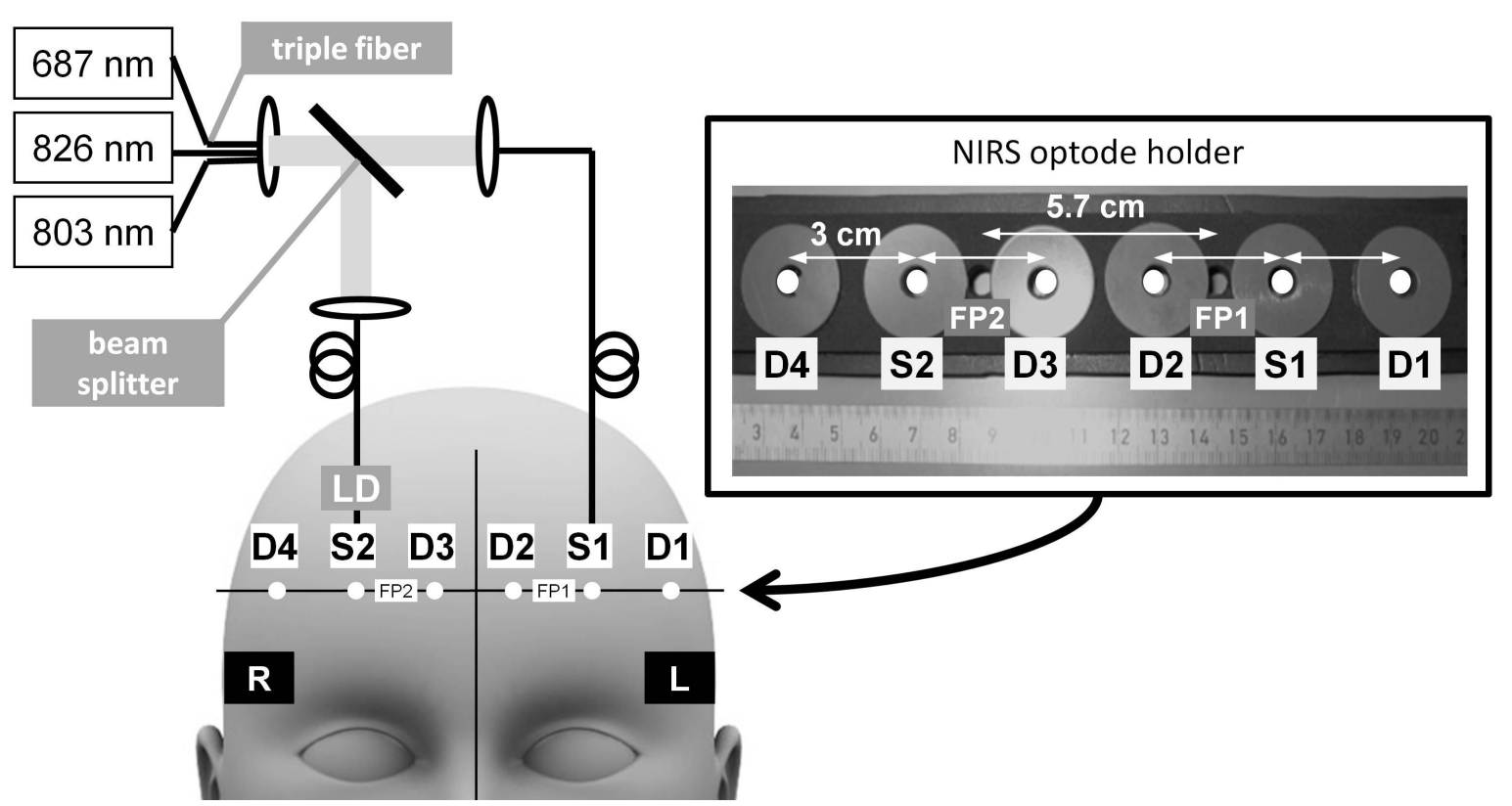

Figure 1. Position of the sources (S1, S2) and the detectors (D1 to D4) on the frontal lobe. The totally available laser light was splitted into light sources S1 and S2. The position of the Laser Doppler probe is denoted by LD. The inset shows the design of the optode holder. It was alligned to the position of FP1 and FP2.

\section{METHODS}

The measurements were performed on 15 healthy subjects ( 5 female, 10 male, mean age 34.9 years, standard deviation 7.2 years). A continuous performance task (CPT) combined with a semantic categorization task was used in order to achieve activation of the frontal lobe (bilateral Brodmann Area 10, BA10). A time series of German words were presented to the subjects with $1 \mathrm{~s}$ presentation time and $1 \mathrm{~s}$ inter-stimulus interval. In the main task (sem-CPT) each of the words represented either a concrete or an abstract concept. The subjects had to press a button whenever they saw a concrete word that followed an abstract one. In the control task (word-CPT) the abstract word was always the same, i.e. VORZUG (German for "preference") followed by the concrete word KOFFER (German for "suitcase"). All in all 18 stimulation and resting periods (34.15 s and $31.15 \mathrm{~s}$ duration, respectively) have been completed by each subject. Each odd stimulation was a word-CPT task whereas each even task was a sem-CPT. A pre- and postbaseline of 2 minutes duration each were recorded. The changes in oxy- and deoxyhemoglobin ( $\mathrm{HbO}, \mathrm{HbR})$ were recorded using the PTB time-domain brain imager [3] at three wavelengths $(687 \mathrm{~nm}, 803 \mathrm{~nm}, 826 \mathrm{~nm})$. The device is equipped with four detectors each connected to a TCSPC module (Becker \& Hickl SPC-134). The placement of all detectors and sources on the forehead is shown in Fig. 1. The laser light was first combined in a triple fiber and then split and coupled into two fibers placed on the left and right side of the frontal lobe. Each source fiber emitted an overall average power of $3 \mathrm{~mW}$. The detection fiber bundles were placed along the frontal lobe, with a source-detector separation of $3 \mathrm{~cm}$. For a reproducible placement the Fp1 and Fp2 positions corresponding to the EEG 10-20 system were first marked on the skin of each subject. Then the optode holder was aligned using these marks and the holes in the holder (Fig.1). Therefore the two central source-detector pairs always covered the Fp1 and Fp2 positions.

We analyzed the time-domain NIRS data in terms of moments [4]. The changes in $\mathrm{HbO}$ and $\mathrm{HbR}$ were calculated from the changes in the light attenuation using the modified Beer-Lambert law [7]. For each subject we calculated the timevariant differential pathlength factor (DPF, as a function of the experiment time) from the mean time of flight of photons through the tissue. In addition relative changes in $\mathrm{HbO}$ and $\mathrm{HbR}$ were obtained from the changes of the variance $V$ of a distribution of times of flight of photons (DTOF). The variance sensitivity factor $V S F$ [4] was assumed to be independent of wavelength but otherwise unknown. Therefore in Fig. 4 we show relative changes of $\mathrm{HbO}$ and $\mathrm{HbR}$ scaled by a factor $k=V S F \cdot \ln (10)$. In both cases the hemoglobin extinction coefficients for the corresponding wavelength from [6] were used to obtain the concentration changes from the changes in the absorption coefficient. The advantage of the variance 
signal is its higher relative sensitivity to absorptions changes in deeper layers compared to the light attenuation signal $[3,4]$.

In addition to the NIRS data, a set of systemic parameters was recorded simultaneously. The mean blood pressure was measured non-invasively by the PortaPres system (TNO TPD Biomedical Instrumentation) on the left hand fingers. A laser Doppler (LD) device (floLAB, Moor Instruments) enabled the measurement of the scalp blood flow (SBF) and scalp red blood cells (RBC) concentration changes. The LD probe was placed on the right hemisphere. The SBF and the RBC signals were normalized to the pre-baseline in order to obtain relative changes. The respiration, GSR, electro-

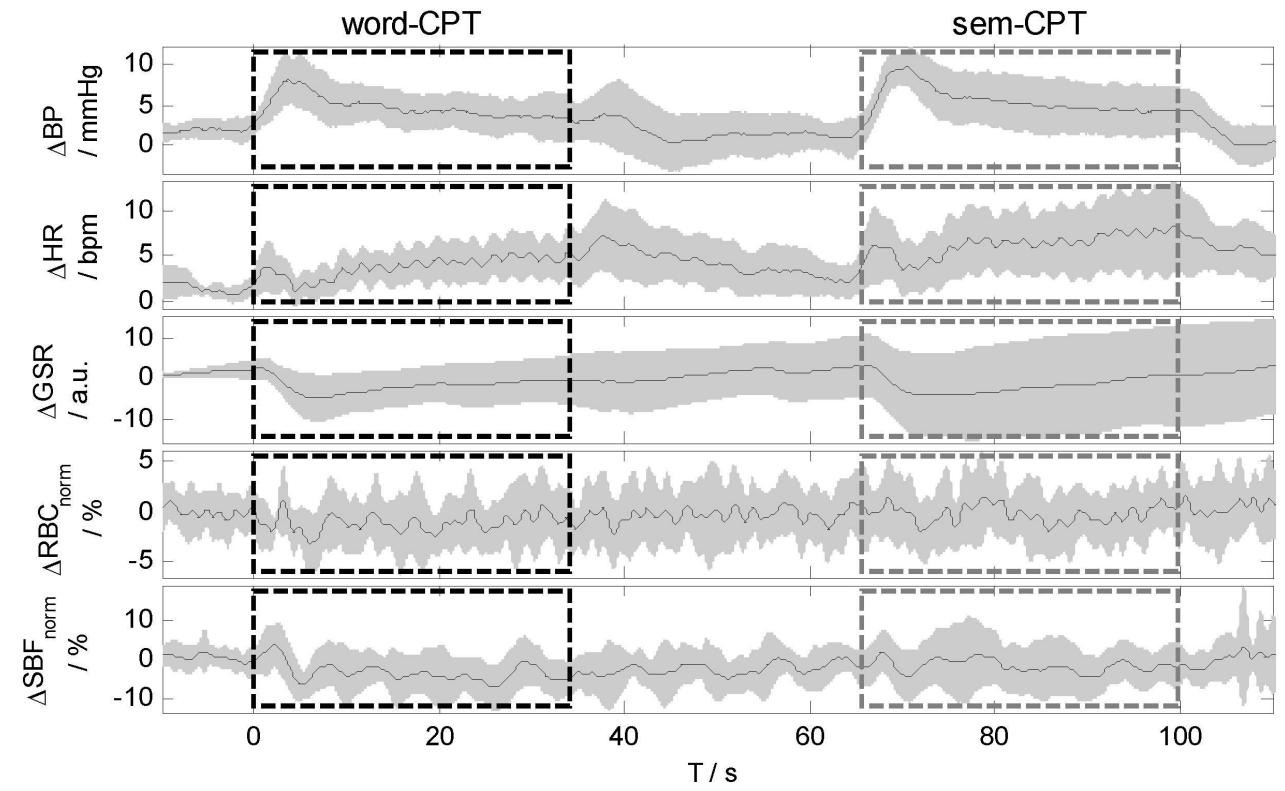

Figure 2. Group average of some physiological signals (BP - mean blood pressure, HR - heart rate in beats per minute, GSR - galvanic skin response, RBC - red blood cells concentration changes, SBF - flux) with the corresponding standard deviation. The rectangles correspond to the control task and the semantic task, respectivly.
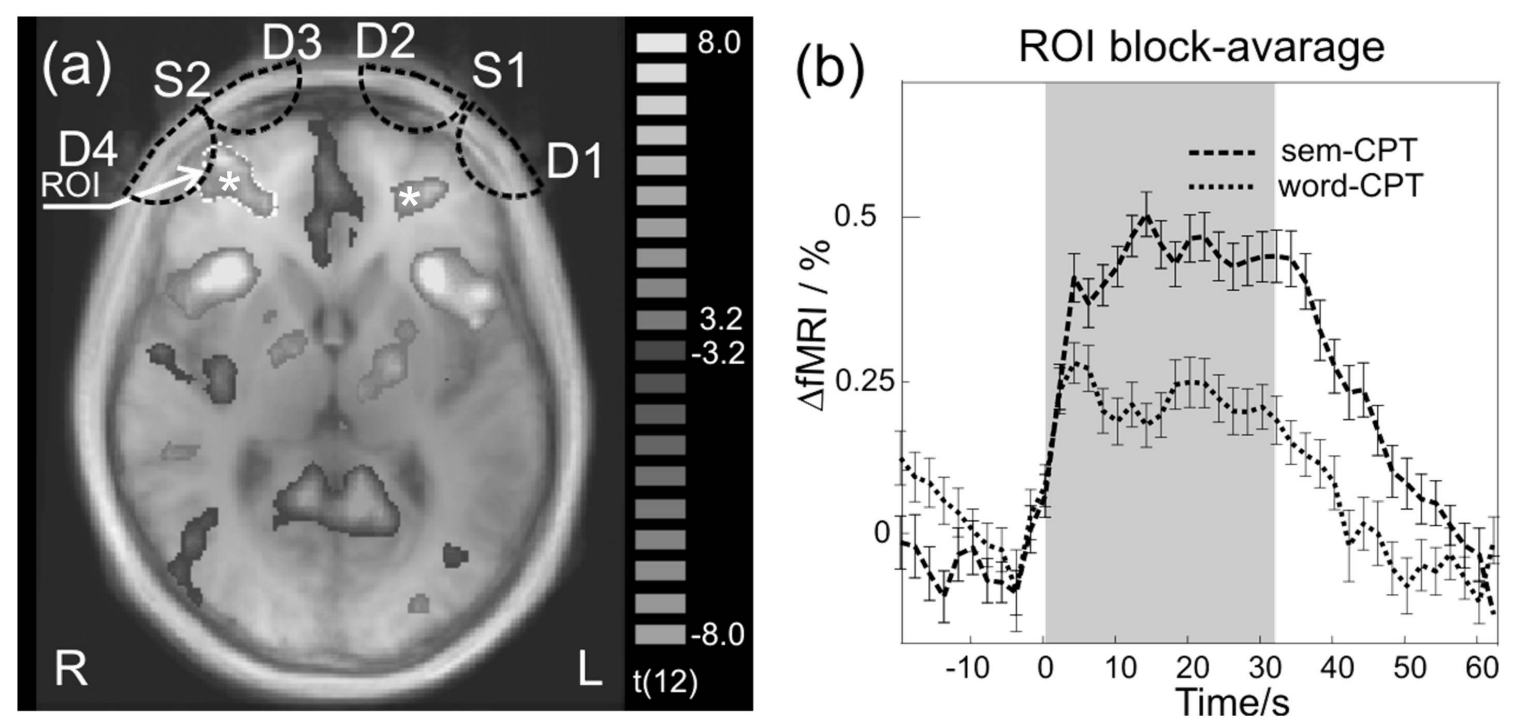

Figure 3. The results of the group analysis of the fMRI experiment (a) the group activation map overlaid on a group anatomical template. The position of the optical sources and detectors is indicated with numbers. The relevant activated regions in bilateral BA 10 are indicated with white asterix. (b) the fMRI signal time course from the Region-Of-Interest in the right BA10 averaged over the whole subject population and all repetitions of the task. 
cardiogram (ECG) as well as the blood volume pulse on the ear were recorded by a separate device (Nexus-10, Mind Media). We used the ECG signal to calculate the heart rate (HR).

\section{RESULTS}

The group-averaged physiological signals are shown in Fig. 2. Mean blood pressure and heart rate show clear stimulation-related changes. Both signals increase with stimulus onset, but the behavior at later times is different. The GSR signal which is related to the sympathetic outflow of the nervous system shows clear task-evoked changes. The observed decrease of the GSR signal corresponds to a decrease in skin resistance, which is induced by a task evoked increase in the sympathetic nervous activity. The changes in SBF and RBC do not show a clear correlation with the task.

The activation map obtained from the group analysis of fMRI data is shown in Fig. 3a. Task-induced bilateral activation in the BA 10 was observed in the area covered by the optical channels 1 and 4 with stronger activation on the right side. The time course of the fMRI signal averaged over subject population and over all repetition of the task blocks is shown on the Fig. 3b. The amplitude of the fMRI signal changes is in the order of $0.2 \%$ which is much lower than what is usually obtained for motor activation $(\sim 4 \%)$. The time course of the fMRI signal in the activated area is in good agreement with the typical hemodynamic response to cerebral activation.

The group-averaged changes in $\mathrm{HbO}$ and $\mathrm{HbR}$ estimated from the td-fNIRS measurements are presented in Fig. 4. Significant task-evoked changes are observed in all four channels and for both hemoglobin species. However, the time course of the signals differs strongly for different channels and between $\mathrm{HbO}$ and $\mathrm{HbR}$. Signal changes similar to a typical cerebral activation can be seen in channel four and less clear in channel one. These findings are in good agreement with the localization of the activation found in the fMRI recordings (see Fig. 3a). The mean concentration changes were found to be in the order of $0.2 \mu \mathrm{M}(\mathrm{HbO})$. This value is smaller than the changes typically found for motor task activation which can reach several $\mu M$ for $\mathrm{HbO}$ [5]. The signals in other channels do not show the typical shape of a cerebral hemodynamic response. On the contrary, they exhibit an increase right after the stimulus onset, a subsequent steady decrease during the stimulus period and a steady increase during the rest period, resembling an approximately triangular shape. No clear baseline during the rest period and no activation plateau can be identified. Therefore we hypothesize that these optical signals are distorted by systemic artifacts which correlate with the stimulus.

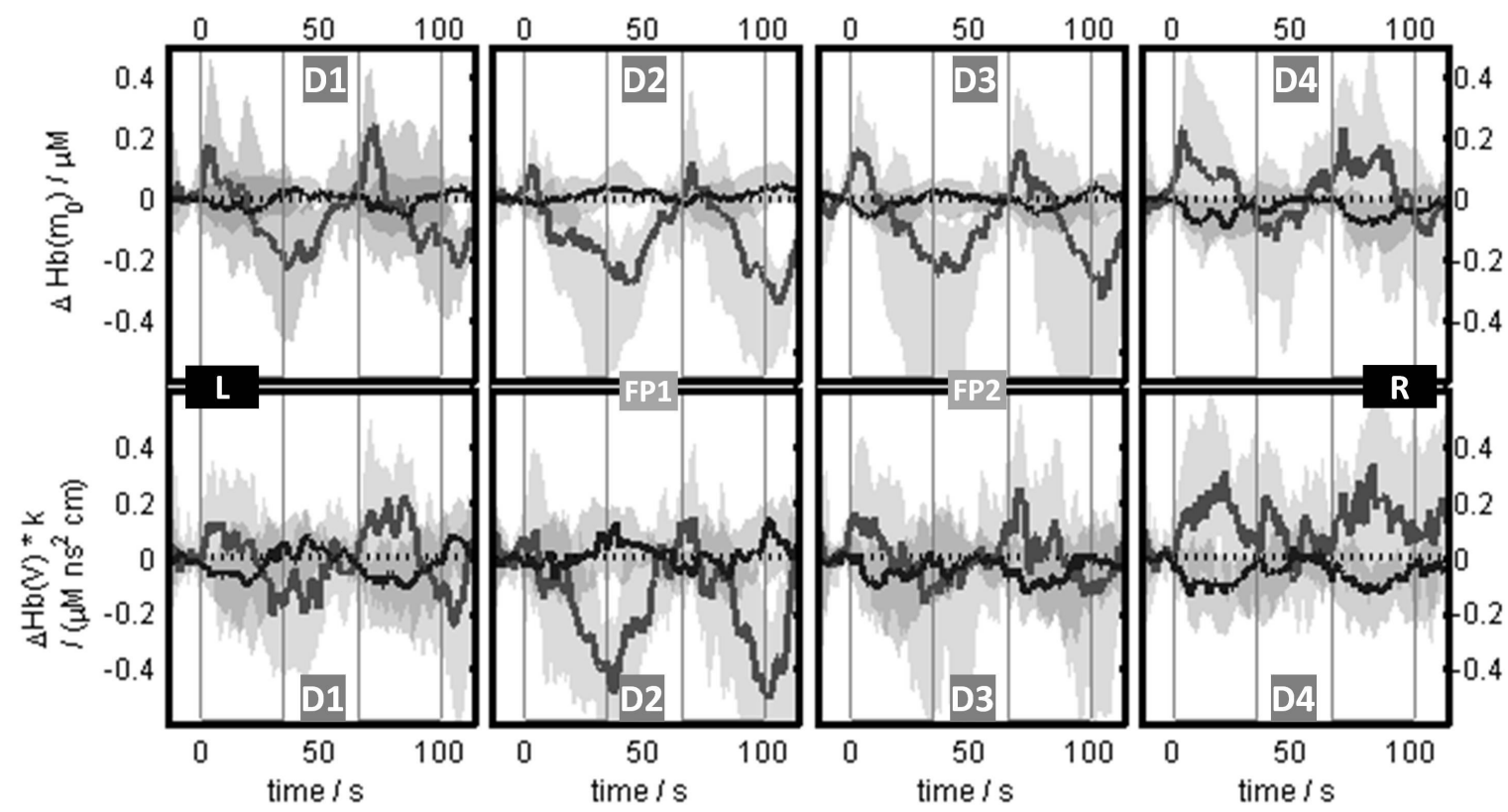

Figure 4. Group average of $\mathrm{HbO}$ (grey) and $\mathrm{HbR}$ (black) concentration changes. The shadowed areas refer to percentiles covering approximately $68 \%$ of the values. The columns correspond to the four detectors (D1 to D4). The changes in $\mathrm{HbO}$ and $\mathrm{HbR}$ in the upper row were calculated from the attenuation changes and individual time-variant DPF. The relative Hb changes in the lower row were calculated from the variance $V$ of DTOFs. The grey rectagles indicate the control (left, from $0 \mathrm{~s}$ to $34 \mathrm{~s}$ ) and semantic (right, from $65 \mathrm{~s}$ to $99 \mathrm{~s}$ ) task. 
Furthermore there are visible differences in the signals obtained from the attenuation changes and changes in the variance. The changes in $\mathrm{HbO}$ obtained from the attenuation clearly show the triangular shape in the first three channels and less clear in channel four. At the same time the variance signal is mainly affected by such triangular component in channels 2 and 3 only. The prominent triangular shape is present in the oxygenated hemoglobin but is not visible in HbR. It is more pronounced in the light attenuation signal compared to the signals derived from variance. This finding is an indication of a superficial origin of the triangular-shaped signal component, having in mind that the depth sensitivity profile for variance peaks at a larger depth compared to the integral [3, 4]. None of the physiological signals shown in Fig. 2 matches the triangular shape. Therefore it cannot be tracked back to a single physiological source in this experiment. It is possible that a combination of several independent systemic physiological signals might explain the distorted shape of the optical signals.

In order to identify the most pronounced influence of systemic physiological signals on changes in oxy- and deoxyhemoglobin we calculated linear correlation coefficients (Pearson's $r$ ) across the acquired signals. The $r$ values were obtained on the individual subject level for all hemoglobin signals ( $\mathrm{HbO}$ and $\mathrm{HbR}$ from $m_{0}$ and $V$ ) and all detectors. The averaged values of correlation coefficients are show in Fig. 5 for three choices of the correlation intervals, (a) word-CPT only, (b) sem-CPT only and (c) both CPT together. The highest average correlation coefficients can be found for mean blood pressure as well as galvanic skin response where the situation is very similar for all $\mathrm{HbO}$ and $\mathrm{HbR}$ signals and all tasks. The different sign for the correlation coefficients found for $\mathrm{HbO}$ and $\mathrm{HbR}$ is a consequence of their direction of change. The SBF signal shows remarkable correlation with the superficial light attenuation signal only and only for

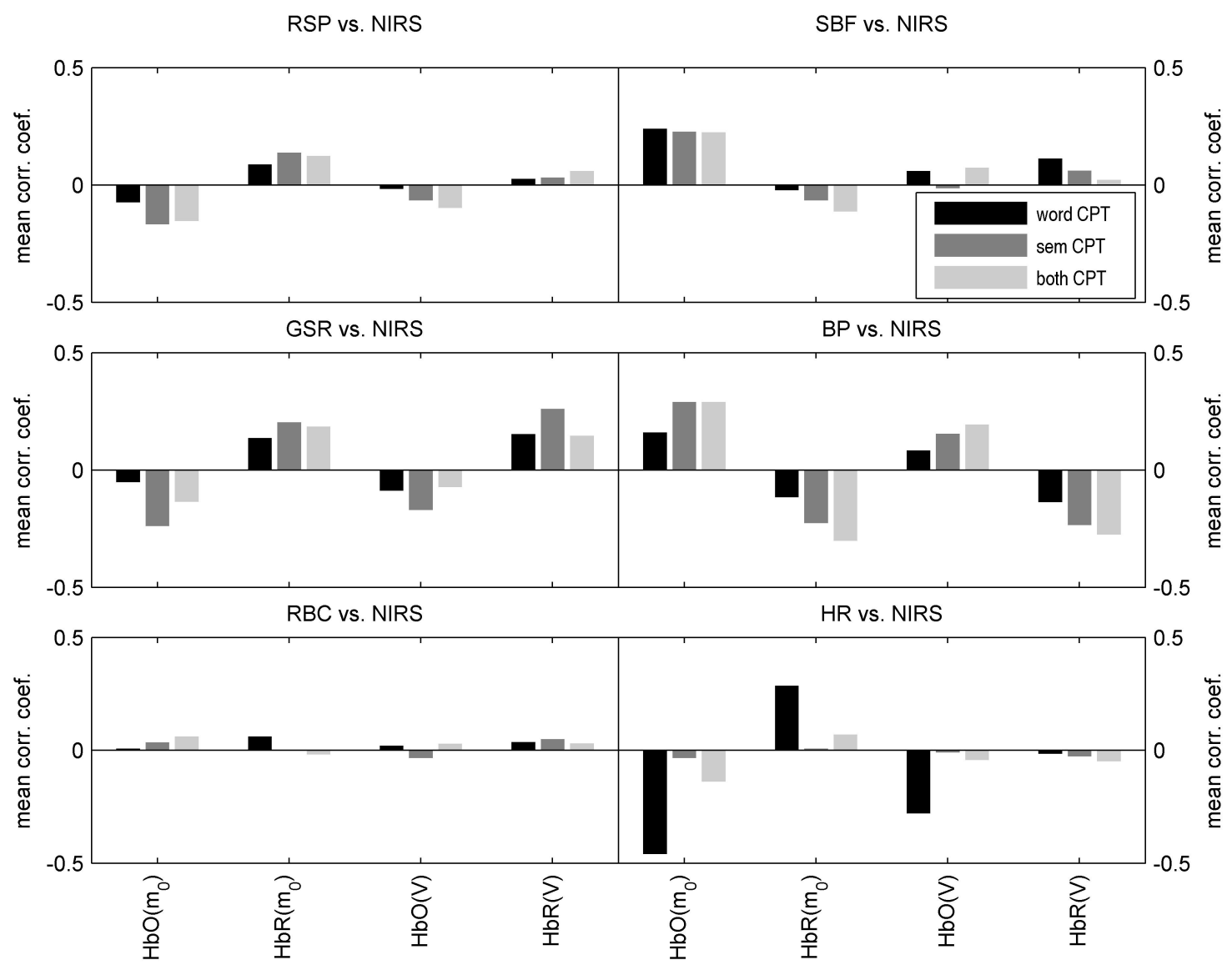

Figure 5. Mean correlation coefficients (group average) calculated between six systemic physiological signals (RSP respiration, $\mathrm{SBF}$ - scalp blood flow, GSR - galvanic skin response, BP - mean blood pressure, RBC - red blood cells concentration changes, HR - heart rate) and the four hemoglobin signals. The calculation was performed for the two tasks (word-CPT and sem-CPT) separately and for both tasks together (both CPT). 
$\mathrm{HbO}$, as expected. An interesting behavior can be observed for heart rate. The correlation is high only for the word-CPT task, the superficial light attenuation signal and $\mathrm{HbO}$. The $\mathrm{HbR}$ signal from the variance is uncorrelated. The respiration signal shows low correlation for the superficial light attenuation signal and none for the variance signal. The RBC signal shows no correlation at all. Altogether the results indicate a complex interplay of systemic physiological signals with the hemoglobin concentration changes. The highest and most stable effects seem to come from the mean blood pressure and galvanic skin response confirming sympathetic and parasympathetic influences. The heart rate shows a selective and task-dependent behavior.

\section{CONCLUSION}

We performed a multi-modality measurement on the frontal lobe during a continuous performance task. Compared to a typical motor task, a rather low activation signal was found in terms of hemoglobin concentration changes. The spatial location of the activation agrees with findings of the fMRI experiment with the same paradigm. The optical signal was found to be seriously distorted by a presumably systemic superficial artifact of a nearly triangular shape that is locked to the stimulation. This artifact was more strongly present in HbO concentration changes and less pronounced in HbR. The correlation analysis of the fNIRS time courses with the time courses of systemic physiological parameters indicated that this artifact might be induced by changes of the systemic blood pressure and the sympathetic outflow. However, none of the investigated systemic physiological signals showed a similar triangular shape. Therefore the distortion of the optical signals cannot be regressed out with the help of a single systemic physiological parameter.

Changes in the mean blood pressure and the sympathetic outflow are concomitant with a wide variety of cognitive and emotional processes. Therefore we expect artifacts similar to those observed in our measurements to be present for many paradigms used in fNIRS studies on the frontal lobe. For that reason special care has to be taken when interpreting brain activation maps and designing fNIRS studies.

\section{ACKNOWLEDGEMENTS}

The research leading to these results has received funding from the European Community's Seventh Framework Programme [FP7/2007-2013] under grant agreement n FP7-HEALTH-F5-2008-201076.

\section{REFERENCES}

[1] Tachtsidis, I., Leung, T. S., Devoto, L., Delpy, D. T., Elwell, C. E., "Measurement of frontal lobe functional activation and related systemic effects: a near-infrared spectroscopy investigation" Adv Exp Med Biol. 614, 397-403 (2008).

[2] Tachtsidis, I., Leung, T. S., Chopra, A., Koh, P. H., Reid, C. B., Elwell, C.E., "False positives in functional near-infrared topography," Adv Exp Med Biol. 645, 307-314 (2009).

[3] Wabnitz, H., Moeller, M., Liebert, A., Walter, A., Erdmann, R., Raitza, O., Drenckhahn, C., Dreier, J. P. , Obrig, H., Steinbrink, J., Macdonald, R., "A time-domain NIR brain imager applied in functional stimulation experiments," in Photon Migration and Diffuse-Light Imaging II Proc. OSA-SPIE Biomed. Opt. 5859, 70-78 (2005).

[4] Liebert, A., Wabnitz, H., Steinbrink, J., Obrig, H., Möller, M., Macdonald, R., Villringer, A., Rinneberg, H., "Time-Resolved Multidistance Near-Infrared Spectroscopy of the Adult Head: Intracerebral and Extracerebral Absorption Changes from Moments of Distribution of Times of Flight of Photons," Appl. Opt. 43, 3037-3047 (2004).

[5] Colier, W. N. J. M., Quaresima, V., Oeseburg, B., Ferrari, M. , "Human motor-cortex oxygenation changes induced by cyclic coupled movements of hand and foot," Exp. Brain Res. 129, 457-461 (1999).

[6] Matcher, S. J., Cope, M., and Delpy, D. T.,. "Use of the water absorption spectrum to quantify tissue chromophore concentration changes in near infrared spectroscopy," Physics in Medicine \& Biology 39, 177-196 (1994).

[7] Delpy, D.T., Cope, M., van der Zee, P., Arridge, S., Wray, S. and Wyatt, J., "Estimation of optical pathlength through tissue from direct time of flight measurement," Phys. Med. Biol. 33, 1433-42 (1988). 